\title{
An Energy Efficiency Routing Algorithm of Wireless Sensor Network Based on Round Model
}

\author{
Zhang Ying-Hui \\ Software Center of Northeastern University, Shenyang, China \\ zhangyh_neusoft@163.com
}

Keywords: Wireless Sensor Network; Energy; Routing.

\begin{abstract}
In order to improve the energy efficiency of wireless sensor network in large area, this paper proposes an energy efficiency routing algorithm of wireless sensor network based on round model. Our algorithm uses the method of split round to achieve multi-hop communication between cluster heads. By building clusters with different size in the different round, it can solve the hot spots problems in sensor network. Under different cluster head election strategies, it can satisfy the two scenarios of node energy homogeneous and heterogeneous. The simulation results show that on the large area environment, our algorithm can greatly balance node energy consumption, prolong the lifetime of the network.
\end{abstract}

\section{Introduction}

High efficient routing algorithm can greatly improve the energy efficiency of wireless sensor network. The clustering routing algorithm is high efficient algorithm which is suitable for the characteristics of sensor network [1,2]. Heinzelman has proposed the low energy adaptive clustering hierarchy algorithm (LEACH) [3]. Its main idea is to let each nodes act as cluster heads in turns, and make the network energy consumption uniform, reduce the energy waste of network's failure time. LEACH algorithm only considers the single hop model, so it is only suitable for small WSN network. Reference [4] proposed the M-LEACH algorithm (multi-hop variant of LEACH), nodes in the cluster transmit data to the cluster head by other node in the cluster, and does not use the single hop way. Reference [5] put forward a multi-hop cluster head model, and adopt the strategy to generate the cluster head of each layer from bottom to top, and get a multi-layer structure of WSN network in the end. Reference [6] use the objective function based on the cost to analyze the design of sensor network, and deduce the suitable scope of single hop and multi-hop, and then finally put forward a kind of single hop and multi hop hybrid routing algorithm, which can overcome the hot spot problem in the network. Reference [7] proposed the energy-efficient unequal clustering algorithm (EEUC). In EEUC algorithm, each node determine whether or not it become the pseudo cluster head randomly according to a predetermined threshold, the pseudo cluster head node calculates the distance to the sink according to the energy of received signal, and competition area with different radius is formed. The pseudo cluster head with maximum energy become the cluster head within the region by competition. EEUC algorithm is more stable than LEACH, its energy consumption is more even than LEACH, and the life of network is longer.

This paper put forward the multi-hop clustering routing algorithm based on round model (EMHA). It firstly divides the monitor area into multi-concentric round, the outer round cluster heads forward data by the cluster head in inner round, and makes the generation algorithm of multiple hops routing simple and clear. Then it is aim to minimize energy consumption in the first round, and deduce the optimal first round cluster number; Then it use the principle of uniform energy consumption, and deduce the optimal number of cluster heads of rest round, finally form a uniform energy consumption, multi-hop WSN network with heterogeneous cluster size, and overcome the hot spot problem in the network. The algorithm completely avoids the dependent on single hop; the randomly selected cluster heads can overcome the drawback of cluster head certainty distribution, so it can be truly applied in the large area environment. 


\section{Description of algorithm}

Assume $N$ nodes are distributed in the circle region with radius $R$, the node's location is known, and sink node is located in the middle of the region. Because the nodes are uniform distributed in the circular region with a radius $R$, and the randomly generated cluster head on the basis of them also must be evenly distributed in the area. In order to facilitate the establishment of the multiple hops path and the related parameter analysis, we logically divide the entire region into $S$ uniform spacing of concentric circle area, the center of the circle is Sink node, and the spacing between the circles is $\delta$.

The rounds are numbered as round 1 , round $2, \ldots$, round $S$ from sink node outwardly. In order to facilitate the node's configuration and deployment, this algorithm only deployed the general information set I about the area in the sink node, which is defined as $I=\{R, N, S\}$ (1).

After nodes have been deployed, the sink node calculated the optimal initial cluster head election probability $\mathrm{T}=\left\{\mathrm{T}_{k} \mid k \in 1,2, \ldots S\right\}$ of each round according to the general information $\mathrm{I}$, and then packaged I, T and the position pos $_{\text {sink }}$ of the sink node as SINK_ADV_SYS_MSG message to broadcast in the area. When the area is large, SINK_ADV_SYS_MSG would be flood broadcasted, every node which firstly received the package would broadcast the message by once time, and make the message can reach each node in the area. After any node $j$ in the area has received the broadcast package, it can determine its round according to the overall information I and its own position pos namely, $k$ can be determined, if $(k-1) \delta<d\left(\operatorname{pos}_{j}, \operatorname{pos}_{\sin k}\right)<k \delta, j$ is in the $k^{\text {th }}$ round, $k=1,2, \ldots \mathrm{S}(2)$.

Each node in the round makes an independent cluster head election according to the cluster head election probability of its round. The elected cluster head would outwardly notice CH_ADV_MSG message, the message contains the location of the cluster head. The around cluster heads and ordinary nodes would receive the message. After the cluster head messages has been noticed, ordinary nodes select the nearest cluster heads from the received CH_ADV_MSG message of multiple cluster heads, and send NODE_JOIN_MSG messages to it. The cluster head nodes of the $k+1$ round choose its nearest cluster heads as the next hop from all the received CH_ADV_MSG message of all the $k^{\text {th }}$ cluster head, and send RELAY_REQ_MSG to it, thus formed the corresponding multiple hops routing between clusters. The cluster head notify adopt the broadcast way, for some factors such as access conflict and interferences, there may be a little amount of nodes (cluster head) have not received any $\mathrm{CH} \_\mathrm{ADV} \_$MSG message. $\mathrm{LEACH}$ algorithm make this kind of node send data directly to the sink node, which is low efficiency or impossible in the larger area; In addition, for the cluster head node which have not receive the announcement information of cluster heads, it can cause the communication cannot be established. For this reason, after the announcement of cluster head is over, the nodes which have not received any CH_ADV_MSG message would actively outward CH_REQ_MSG message, the message contains its own ID. The corresponding cluster head which have received the message sent CH_ACK_MSG message. Through this process, it can reliably building cluster and multiple hops routing.

For the data correlation from different clusters is small, the polymerization efficiency is not high. The cluster head don't process the forwarding data packets from the cluster head, it just forward to its next hop. In such clustering multi-hop network, the forwarding load of cluster node in the round which is nearest to the sink node is larger, it is easy to run out of energy and dead in advance, and this is the hot spots problem in wireless sensor network. Our algorithm builds clusters with different size in different rounds by controlling cluster head election probability of different rounds, $T_{k}, k=1,2, \ldots$, and balance the energy consumption between cluster heads of different rounds. In general, it make the formation size of cluster in the round whose distance is closer to sink node is smaller, the energy consumption of the cluster heads in communications within cluster and the data aggregation is less, so as to compensate its energy consumption for forwarding the data packages from the outer round cluster node. And the formation size of cluster in the round whose distance is farther to sink node is larger, and make the energy consumption of communication within the cluster and data aggregation increases, so as to offset the save energy for forwarding less packets. The average cluster size of round $k$ is controlled by the cluster head of round $m_{k}, k=1,2, \ldots$ S. If we obtain the optimal cluster head of a round, then we get the optimal cluster head election probability of the round. 


\section{Realization process of algorithm}

EMHA algorithm is described as follows:

(1)Sink node calculated the optimal cluster head election probability $T$ of each round according to the overall information collection $I, T$ and the position of Sink node as SINK_ADV_SYS_MSG messages, flood broadcast them in the network;

(2)All nodes use formula (2) to determine their rounds, and find the cluster head election probability from SINK_ADV_SYS_MSG package;

(3)Select cluster heads: each node generated a random number by the uniform distribution, and compared it with the cluster head election probability of its rounds, and determined whether it is as the cluster head in this round;

(4)When the elected cluster head broadcast CH_ADV_MSG message with radius $r=2 \delta$, the message contained the cluster head's ID and position;

(5)Non-cluster head node selected the nearest cluster heads to join according to the received CH_ADV_MSG messages, and send the join request message NODE_JION_MSG to cluster head node, the content of the message is the node's ID; The $k+1$ round cluster head selected its nearest cluster heads as the next hop according to the received notification message of the $\mathrm{k}^{\text {th }}$ round cluster head, and send its forwarding request message RELAY_REQ_MSG, the content of the message is their own ID and the serial number of round;

(6)Th node and the cluster head node which have not received any of the notification messages actively broadcast CH_REQ_MSG message, the message contains its own ID; the cluster head node which has received the message sent $\mathrm{CH}$ _ACK_MSG message to them, the message contained the cluster head's ID. Thus each ordinary node found its belonged cluster, each cluster head find his next hop. Then the cluster and multiple hops routing building phase is over, it has entered into the data collection phase;

(7)Each node makes a cluster head rotation per $t$ second, and cycle the process from (3) to (7).

\section{Experiment}

The simulation algorithm includes LEACH, EEUC, and EMHA. The simulation environment use NS2. The goal of simulation is different life cycles and energy efficiency of algorithm. We make the simulation on scenarios whose radius are from $40 \mathrm{~m}$ to $720 \mathrm{~m}$, and the total number of nodes are $\mathrm{N}=400, \mathrm{~N}=1000, \mathrm{~N}=1600, \mathrm{~N}=8000$. In order to ensure that nodes can communicate for a relatively long time in large area, in NS2, the initial energy of each ordinary node is $10 \mathrm{~J}$, the initial energy of each senior node is $20 \mathrm{~J}$, the ratio of senior node is $10 \%$ or zero, senior node and common node are random and uniform distributed in the area. For the algorithms which are analyzed and compared in this thesis are improved or expanded based on LEACH algorithm, so various public parameters in our simulation environment are selected according to the parameters in LEACH algorithm. The main parameters in the simulation are shown as Table 1.

Table 1 Parameters of simulation

\begin{tabular}{|c|c|}
\hline Parameter name & Value \\
\hline broadband & $1 * 10^{5} \mathrm{Mbit} / \mathrm{s}$ \\
\hline$E_{\text {elec }}$ & $30^{*} 10^{-8} \mathrm{~J} / \mathrm{bit}$ \\
\hline$\varepsilon_{f s}$ & $10 \mathrm{pJ} / \mathrm{bit} / \mathrm{m}^{2}$ \\
\hline$E_{m p}$ & $0.001 \mathrm{pJ} / \mathrm{bit} / \mathrm{m}^{4}$ \\
\hline$E_{D A}$ & $3 n J / \mathrm{bit} / \mathrm{signal}$ \\
\hline$d_{o}$ & $100 \mathrm{~m}$ \\
\hline Queue length & 100 packets \\
\hline Delay of link & $15 \mathrm{us}$ \\
\hline Cost of packet head & 20 byte \\
\hline Size of data packet & 400 byte \\
\hline
\end{tabular}


Figure 1 is the residual energy distribution at the end of lifetime, we can find that LEACH algorithm has many nodes with larger residual energy, and the nodes with smaller residual energy only occupy a small proportion, which means that when the area is larger, LEACH algorithm cannot ensure uniform consumption of energy. In EMHA algorithm, the nodes with the residual energy around 1.5 occupy the largest proportion, and it is closer to zero than LEACH algorithm, and the curve of tail is shorter, it shows that its energy utilization efficiency is much better than SEP. We can also know from the figure that the residual energy of EEUC algorithm is more close to zero; this is because the multi-hop EEUC algorithm uses the node's residual energy as an important indicator to select the next-hop of cluster head, which can promote the equalization of energy consumption.

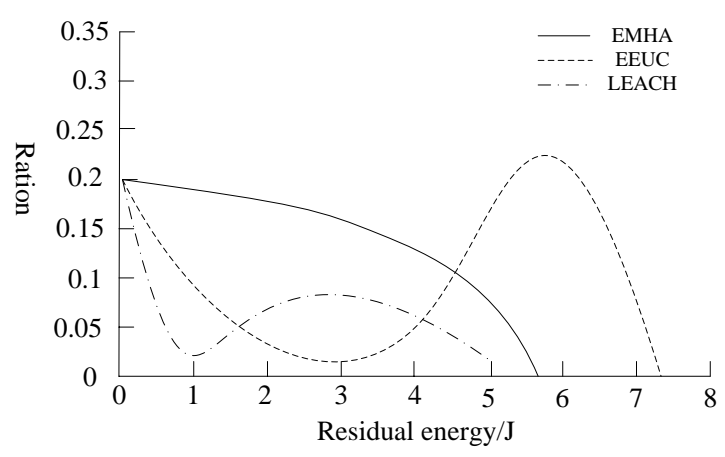

Fig.1 Residual energy distribution

Figure 2 shows that the average lifetime of LEACH algorithm is 161s. This is because when the node's energy is same, LEACH algorithm in fact equivalent to EEUC algorithm. EEUC algorithm has four adjustable parameters, our simulation uses $T=0.4, R_{\text {comp }}=90 \mathrm{~m}, \mathrm{c}=0.5$, TD_MAX $=150 \mathrm{~m}$, the average lifetime is 301s. Thus the average lifetime of EMHA is longer than LEACH, and EEUC. It shows that when nodes are density, the network area is larger, the energy consumption of LEACH algorithm is large by using single hop, and shortens the network lifetime; But EMHA has small signal transmission radius, the conflict range between nodes is small, energy consumption is relatively uniform and greatly improve the energy utilization efficiency, and greatly prolong the lifetime.

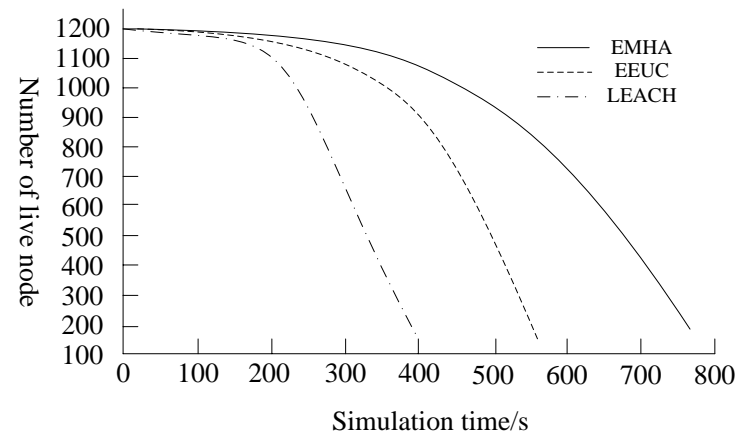

Fig.2 Comprasion of lifetime

\section{Summary}

The EMHA algorithm use round mode to realize the multi-hop routing of network, and make the cluster which is close sink node is small and the cluster which is farther to sink is larger, so it can better overcome the hot spot problem in the network. The node in the network only needs to know its position and the location of sink node, and don't need to know the global network information, which brings convenience for practical applications. EMHA can adapt to the situations of energy homogeneous and heterogeneous, it has more extensive applicability. Simulation shows that under the situation of large area, the network life cycle of our algorithm is loner than one hop algorithm LEACH, and multi-hop algorithm EEUC, energy consumption is more evenly. 


\section{References}

[1] MHATRE V, ROSENBERG C, KOFMAN D, et al. A minimum cost heterogeneous sensor network with a lifetime constraint, IEEE Transactions on Mobile Computing, Vol.4,2011, pp.4-15.

[2] ORO S, HEINZELMAN W B. Prolonging the lifetime of wireless sensor networks via unequal clustering, Journal of network, Vol.3, 2012, pp.153-161.

[3] HEINZELMAN W R, CHANDRAKASAN A P. An application-specific protocol architecture for wireless micro-sensor networks, IEEE Transactions on Wireless Communications, Vol.1, 2002, pp.660-670.

[4] BANDYOPADHYAY S, COYLE E J. An energy efficient hierarchical clustering algorithm for wireless sensor networks, Proceeding of INFOCOM, Vol.1, 2008, pp.1713-1723.

[5] MHATRE V, ROSENBERG C. Homogeneous vs. heterogeneous clustered sensor networks: a comparative study, Proceedings of IEEE International Conference on Communications, Vol.3, 2004, pp. 3646-3651.

[6] MHATRE V, ROSENBERG C. Design guidelines for wireless sensor networks: communication, clustering and aggregation, Journal of Ad hoc Networks, Vol.2, 2012, pp. 45-63.

[7] LI C F, YE M, CHEN G. An energy-efficient unequal clustering mechanism for wireless sensor networks, Proceedings of IEEE International Conference on Mobile Ad hoc and Sensor System, Vol.3, 2005, pp.574-583. 